\title{
Análisis de la variable acoso escolar: Un aporte desde la producción científica latinoamericana
}

\section{Analysis of the Bullying: A Contribution from Latin American Scientific Production}

César Andrés Borja Villanueva*

Universidad Privada Juan Pablo II, Lima, Perú

ORCID: https://orcid.org/0000-0002-4274-4978

Christian Gómez Carrión

Universidad Privada Juan Pablo II, Lima, Perú

ORCID: https://orcid.org/0000-0001-9698-3176

Marya Graciela Barzola Loayza

Universidad Nacional Mayor de San Marcos, Lima, Perú

ORCID: https://orcid.org/0000-0002-1763-4857

Selene Malca Hernandez

Universidad Peruana Los Andes, Huancayo, Perú

ORCID: https://orcid.org/0000-0002-2434-2529

Erika Ruth Alvarado Muñoz

Universidad Privada Juan Pablo II, Lima, Perú

ORCID: https://orcid.org/0000-0003-0959-7117

Ana María Vilchez Huerto

Universidad Nacional Mayor de San Marcos, Lima, Perú

ORCID: https://orcid.org/0000-0003-4666-3830

\section{Francis Díaz-Flores}

Universidad Nacional Mayor de San Marcos, Lima, Perú

ORCID: https://orcid.org/0000-0002-9272-9995

Recibido 15-09-18 Revisado 26-11-19 Aprobado 12-02-20 En línea 03-02-20

*Correspondencia

Email: abv1979@gmail.com
Citar como:

Borja, C., Gómez, C., Barzola, M., Malca, S., Alvarado, E., Vilchez, A., Díaz-Flores, F. (2020). Análisis de la variable acoso escolar: Un aporte desde la producción científica latinoamericana. Propósitos y Representaciones 8(2), e451, doi: http://dx.doi.org/10.20511/pyr2020.v8n2.451 


\section{Resumen}

El estudio describe las características de producción científica latinoamericana sobre la variable acosos escolar en revistas indizadas en Scopus, durante el periodo 2010-2018. Se realiza un estudio descriptivo retrospectivo analizando 637 artículos. Se encuentra que los países que mayor producción tienen son Brasil, Chile, México y Colombia. La mayoría de los artículos se encuentran publicados en las revistas Ciencia e Saude Coletiva y Psicologia Escolar e Educacional. El $90.3 \%$ de las publicaciones representa a artículos originales. Se concluye que la producción científica latinoamericana sobre acoso escolar ha ido aumentando progresivamente durante los últimos años; sin embrago, aún representa un mínimo porcentaje a nivel mundial, es necesario seguir fortaleciendo la generación de difusión de estudios científicos.

Palabras clave: Acoso escolar; Producción Científica; Latinoamérica; Artículos de investigación.

\section{Summary}

The study describes the characteristics of Latin American scientific production on the bullying variable in journals indexed in Scopus, during the period 2010-2018. A retrospective descriptive study is carried out analyzing 637 articles. It is found that the countries with the greatest production are Brazil, Chile, Mexico and Colombia. Most of the articles are published in the journals Science e Saude Coletiva y Psicologia Escolar e Educativo. $90.3 \%$ of the publications represent original articles. It is concluded that Latin American scientific production on bullying has been increasing progressively in recent years; However, it still represents a minimum percentage worldwide, it is necessary to continue strengthening the dissemination of scientific studies.

Keywords: Bullying; Scientific Production; Latin America; Research Articles.

\section{Introducción}

El acoso escolar conocidos en su expresión inglesa como bullying actualmente es considerada un problema de salud pública (Craig, et al., 2009). Las primeras investigaciones se iniciaron en la década de los setenta del siglo XX (Olweus, 1978), permitiendo conceptualizarlo como un tipo específico de agresión relacional (Smith, 2016; ). Por otro lado, Sanmartín (2006) menciona que el acoso escolar se ha convertido en un problema de preocupación creciente, definida como aquel comportamiento violento en centros de enseñanza donde se causa daño físico y psicológico a otros alumnos. Este tipo de violencia expresa conductas como manifestaciones de burlas, lenguaje obsceno, peleas, actos de vandalismo que recibe un niño por parte de otros compañeros que se comportan con él cruelmente, con el objeto de someterlo, asustarlo, amenazarlo atentando contra la dignidad del niño. (Basile, 2004; Piñuel y Oñate; 2007 Hernanadez \& Saravia, 2017)

Investigaciones recientes sugieren una prevalencia de acosos escolar de 29.2\% para Europa y Estados Unidos (Chester, Callaghan, Cosma, Donnelly, Craig, Walsh \& Molcho, 2015), valores entre 20 y $40 \%$ para Estados Unidos (Tokunaga, 2010).). Para Latinoamérica, un informe de la Unicef sobre la violencia escolar en América Latina y el Caribe sugiere que entre 50 y $70 \%$ de los estudiantes han estado involucrados en algún tipo de agresión entre iguales (Eljach, 2011). los estudios a nivel mundial indican que uno de cada tres niños está involucrado en alguna forma de bullying y (Hamm et al., 2015; Zych, Ortega-Ruiz \& Del Rey, 2015). Un análisis realizado en estudios empíricos en contextos escolares de 4 países de América Latina en los últimos diez años revela el predominio de investigaciones que abordan aspectos de la convivencia escolar en prácticas convencionales más que sobre programas de intervención, lo cual puede estar indicando dos cosas: el déficit de estudio de 
experiencias de intervención sobre convivencia escolar, o que estas son aún pocas en la región (Díaz \& Sime, 2016).

Las revisiones sistemáticas o estudios bibliométricos referidos al avance de la investigación de los fenómenos sociales y de salud pública son escasos en Latinoamérica. Pigozi y Machado (2015), quienes tras la revisión de 25 artículos publicados solo en Brasil, reconocen mayormente trabajos de prevalencia. Por otra parte, Herrera-López, Romera y Ortega-Ruiz, (2018) indican que la productividad científica sobre acoso escolar se limita a los últimos 11 años, esto sugiere un bajo aporte al cuerpo teórico científico, pues tras casi 40 años de historia sobre su investigación, solo en la última década aparecen estudios realizados en Latinoamérica.

Por lo anterior, y después de casi cuatro décadas de investigación mundial sobre el acoso escolar, consideramos necesaria la realización de este estudio que tuvo como objetivos identificar el estado actual de la producción científica de esta variable en Latinoamérica, a partir de la revisión de los artículos publicados y disponibles en Scopus.

\section{Método}

Se analizaron 637 artículos que reportaban resultados de investigaciones realizadas sobre violencia escolar escrita por autores con afiliación a instituciones latinoamericanas y que se encontraron en la base de datos Scopus, se escogió ésta por ser una de las más grande y reconocida de resúmenes y referencias bibliográficas de literatura científica. Esta búsqueda y análisis se realizó en setiembre de 2018 y abarcó el periodo 2010-2018. Se utilizó una matriz de registro documental en base a la revisión detallada de cada uno de los artículos, en la que se registró: autores, título del artículo, año de publicación, idioma(s), nombre, URL y/o DOI de publicación.

Este estudio es de tipo descriptivo a partir de la revisión de documentos. Inicialmente se realizó la búsqueda de artículos utilizando palabras clave como: bullying, cyberbullying, intimidación escolar, acoso escolar, ciberacoso, intimidación cibernética, acoso electrónico, intimidación en Internet, acoso en Internet y acoso en línea, se exploró en los títulos/resúmenes/palabras clave, con el fin de recoger todas las opciones posibles.

\section{Resultados}

Con respecto al número de publicaciones por año, se observa un aumento de investigaciones gradual, evidenciando un incremento mayor en el $2018(n=168)$, ver figura 1.



Figura 1. Histórico de producción científica sobre Acosos escolar en Scopus. (2010-2018)

El $90.3 \%$ de tipos documentales correspondió a artículos de investigación, seguido de artículos de revisión con un $4.7 \%$ (ver tabla 1). 
Tabla 1.

Tipo documental de publicaciones sobre acosos escolar en Scopus. (2010-2018)

\begin{tabular}{lll}
\hline Tipo documental & $\mathbf{N}$ & $\mathbf{\%}$ \\
\hline Artículos de investigación & 575 & 90.3 \\
Revisiones & 30 & 4.7 \\
Capítulo de libro & 16 & 2.5 \\
Artículos de conferencia & 11 & 1.7 \\
Carta al editor & 3 & 0.5 \\
Editorial & 2 & 0.3 \\
Total & 637 & 100.0 \\
\hline
\end{tabular}

Los artículos analizados fueron publicados en 160 revistas. La Tabla 2 muestra las revistas donde se han publicado mayoritariamente los artículos con referencia al acoso escolar, el $4.08 \%$ de las publicaciones, se encuentran en la Revista Ciencia E Saude Coletiva, seguido de la Revista Psicologia Escolar E Educacional (3.77\%).

Tabla 2.

Revistas indizadas en Scopus donde se ha publicado artículos referentes a acosos escolar (2010-2018)

\begin{tabular}{|c|c|c|c|}
\hline Revista & $\begin{array}{l}\text { Artículos } \\
\text { publicados }\end{array}$ & $\%$ & $\begin{array}{l}\text { H Index } \\
\text { SJR }\end{array}$ \\
\hline Ciencia E Saude Coletiva & 26 & 4.08 & 39 \\
\hline Psicologia Escolar E Educacional & 24 & 3.77 & 6 \\
\hline Universitas Psychologica & 18 & 2.83 & 18 \\
\hline Frontiers In Psychology & 14 & 2.20 & 81 \\
\hline Revista Brasileira De Epidemiologia & 10 & 1.57 & 26 \\
\hline Revista Mexicana De Investigacion Educativa & 10 & 1.57 & 8 \\
\hline Jornal De Pediatria & 9 & 1.41 & 48 \\
\hline Psykhe & 9 & 1.41 & 14 \\
\hline Revista Medica De Chile & 9 & 1.41 & 34 \\
\hline Revista Paulista De Pediatria & 9 & 1.41 & 14 \\
\hline Cadernos De Saude Publica & 8 & 1.26 & 66 \\
\hline Adolescencia E Saude & 7 & 1.10 & 4 \\
\hline Interamerican Journal Of Psychology & 7 & 1.10 & 16 \\
\hline Psicologia Reflexao E Critica & 7 & 1.10 & 18 \\
\hline Revista Electronica De Investigacion Educativa & 7 & 1.10 & 9 \\
\hline $\begin{array}{l}\text { Revista Iberoamericana De Diagnostico Y } \\
\text { Evaluacion Psicologica }\end{array}$ & 7 & 1.10 & 10 \\
\hline Temas Em Psicologia & 7 & 1.10 & 4 \\
\hline Texto E Contexto Enfermagem & 7 & 1.10 & 16 \\
\hline Psychology Society And Education & 6 & 0.94 & 6 \\
\hline Revista De Salud Publica & 6 & 0.94 & 21 \\
\hline
\end{tabular}

En relación con la contribución por país, se observa que Brasil tiene la mayor cantidad de autoría $(n=291)$, seguido de Chile $(n=117)$, México $(n=114)$ Y Colombia $(n=74)$ entre otros (ver tabla 3 ). 
Tabla 3.

Afiliación institucional por país según publicaciones sobre acosos escolar en Scopus. (20102018)

\begin{tabular}{|c|c|c|}
\hline País & $\mathrm{N}$ & $\%$ \\
\hline Brasil & 291 & 41.9 \\
\hline Chile & 117 & 16.8 \\
\hline México & 114 & 16.4 \\
\hline Colombia & 74 & 10.6 \\
\hline Argentina & 38 & 5.5 \\
\hline Perú & 25 & 3.6 \\
\hline Ecuador & 6 & 0.9 \\
\hline Venezuela & 6 & 0.9 \\
\hline Puerto Rico & 4 & 0.6 \\
\hline Costa Rica & 3 & 0.4 \\
\hline Cuba & 3 & 0.4 \\
\hline República Dominicana & 3 & 0.4 \\
\hline Paraguay & 3 & 0.4 \\
\hline Guatemala & 2 & 0.3 \\
\hline Panama & 2 & 0.3 \\
\hline Uruguay & 2 & 0.3 \\
\hline Honduras & 1 & 0.1 \\
\hline Nicaragua & 1 & 0.1 \\
\hline & 695 & 100.0 \\
\hline
\end{tabular}

\section{Discusión}

Los hallazgos indican que durante la última década las investigaciones sobre acoso escolar han aumentado, siendo el 2018 un año en el cual se destaca una mayor producción a nivel Latinoamericano, siendo Brasil el país que mayor reconocimiento tiene en cuanto a los estudios que difunde. Zych, Ortega-Ruiz y Marín-López, 2016, concuerda con los mencionado anteriormente, indicando que solo en la última década aparecen estudios realizados en Latinoamérica. Además, Herrera-López, Romera y Ortega-Ruiz, (2018) muestran que Brasil, Colombia, México y Chile son los países que mayor producción tienen respecto a esta variable, es posible que este aspecto esté relacionado con un mayor desarrollo científico e investigativo.

Otro indicador importante es la cantidad de investigaciones que se vienen realizando el $90.3 \%$ de la producción representa a artículos originales, Bauman y Bellmore, 2015, indican que la mayor prevalencia de investigaciones se focaliza en la elaboración de revisiones teóricas, así como en el análisis de factores de riesgo y protección, trabajos que ayudan a divulgar la importancia y necesidad de abordaje de esta problemática.

Los resultados del estudio demuestran el avance de la investigación sobre esta variable; sin embargo, la producción de autores con afiliación latinoamericana es aún limitada. Los esfuerzos deben dirigirse hacia el logro de la visibilidad internacional de contenidos elaborados por autores latinoamericanos, debiendo enfocarse en fortalecer e incentivar la generación y divulgación de los estudios científicos (Hernández, 2018). 


\section{Referencias}

Bauman, S., \& Bellmore, A. (2015). New directions in cyberbullying research”. Journal of School Violence, 14(1), 1-10. doi: https://doi.org/10.1080/15388220.2014.968281

Chester, K., Callaghan, M., Cosma, A., Donnelly, P., Craig, W., Walsh, S., \& Molcho, M. (2015). Cross-national time trends in bullying victimization in 33 countries among children aged 11, 13 and 15 from 2002 to 2010. The European Journal of Public Health, 25 (suppl. 2), 61-64. doi: https://doi.org/10.1093/eurpub/ckv029

Craig, W., Harel-Fisch, Y., Fogel-Grinvald, H., Dostaler, S., Hetland, J., Simons-Morton, B., Molcho, M., de Mato, M., Overpeck, M., Due, P., Pickett, W., HBSC Violence \& Injuries Prevention Focus Group y HBSC Bullying Writing Group (2009). A cross-national profile of bullying and victimization among adolescents in 40 countries. International Journal of Public Health, 54 (suppl. 2), 216-224. doi: https://doi.org/10.1007/s00038009-5413-9

Díaz, S., \& Sime, L. (2016). Convivencia escolar: una revisión de estudios de la educación básica en Latinoamérica. Revista Virtual Universidad Católica del Norte, (49), 125-145.

Eljach, S. (2011). Violencia escolar en América Latina y el Caribe. Superficie y fondo, Panamá: Unicef-Oficina Regional para América Latina y el Caribe.

Hamm, M. Newton, A., Chisholm, A., Shulhan, J., Milne, A., Sundar, P., Ennis, H., Scott, S., \& Hartling, L. (2015). Prevalence and effect of cyberbullying on children and young people: A scoping review of social media studies, JAMA Pediatrics, 169(8), 770-777. doi: https://doi.org/10.1001/jamapediatrics.2015.0944

Hernández R. M., \& Saravia, M. Y. (2016). Generalidades del acoso escolar: Una revisión de conceptos. In Revista de Investigación Apuntes Psicológicos ,1(1). 30-40.

Hernández, R. M. (2018). Producción científica latinoamericana en la revista Anales de Pediatría. Anales de Pediatría, 89 (2), 130-131. doi: https://doi.org/10.1016/j.anpedi.2017.12.012

Herrera-López, M., Romera, E., \& Ortega-Ruiz, R. (2018). Bullying y Cyberbullying en Latinoamérica. Un estudio bibliométrico. Revista mexicana de investigación educativa, 23(76), 125-155.

Olweus, D. (1978). Aggression in the schools. Bullies and whipping boys, Londres: John Wiley y Sons.

Piñuel, I., y Oñate, A. (2007). Mobbing escolar: Violencia y acoso psicológico contra los niños. Barcelona: CEAC.

Pigozi, P., \& Machado, A. (2015). Bullying during adolescence in Brazil: an overview", Ciência Saúde Coletiva, 20(11), 3509-3522. doi: https://doi.org/10.1590/1413812320152011.05292014

Sanmartín, J. (2006). Violencia escolar. En A. Serrano. (Ed.). Acoso y violencia en la escuela (pp. 19-31). Barcelona: Ariel.

Smith, P. (2016). Bullying: Definition, types, causes, consequences and intervention", Social and Personality Psychology Compass, 10(9), 519-532. doi: https://doi.org/10.1111/spc3.12266

Tokunaga, R. (2010). Following you home from school: A critical review and synthesis of research on cyberbullying victimization. Computers in Human Behavior, 26(3), 277-287. doi: https://doi.org/10.1016/j.chb.2009.11.014

Zych, I., Ortega-Ruiz, R., \& Del Rey, R. (2015).Scientific research on bullying and cyberbullying: Where have we been and where are we going. Aggression and Violent Behavior, 24, 188198. doi: https://doi.org/10.1016/j.avb.2015.05.015 